Perspective

\title{
Psychiatric Genetics, Epigenetics, and Cellular Models in Coming Years
}

\author{
Chunyu Liu *, Stephen V. Faraone, Stephen J. Glatt \\ Department of Psychiatry, SUNY Upstate Medical University, Syracuse, NY 13210, USA \\ * Correspondence: Chunyu Liu, Email: liuch@upstate.edu.
}

\begin{abstract}
Psychiatric genetic studies have uncovered hundreds of loci associated with various psychiatric disorders. We take the opportunity to review achievements in the past and provide our view of what is coming in the fields of molecular genetics, epigenetics, and cellular models. We expect that SNP-array and sequencing-based studies of genetic associations will continue to expand, covering more disorders, drug responses, phenotypes, and diverse populations. Epigenetic studies of psychiatric disorders will be another promising field with the growing recognition that environmental factors impact the risk for psychiatric disorders by modulating epigenetic factors. Functional studies of genetic findings will be needed in cellular models to provide important connections between genetic and epigenetic variants and biological phenotypes.
\end{abstract}

KEYWORDS: psychiatry; genetics; epigenetics; behavior; population diversity; function study; drug response; cellular phenotype; molecular phenotype; regulatory network

\section{INTRODUCTION}

Molecular genetic research into psychiatric disorders has made substantial progress in the last several decades, delivering important findings about the genetic risks of several conditions. Fast-evolving genotyping and sequencing technologies have driven this progress. The most important findings came from genome-wide association studies

\section{G Open Access}

Received: 28 April 2019

Accepted: 16 August 2019

Published: 22 August 2019

Copyright $@ 2019$ by the author(s). Licensee Hapres, London, United Kingdom. This is an open access article distributed under the terms and conditions of Creative Commons Attribution 4.0 International License. (GWASs), whole-exome sequencing (WES), and whole-genome sequencing (WGS) of various disorders, behavioral traits, and other phenotypes. GWASs has produced the majority of findings in the past ten years [1]. We have learned that common single nucleotide polymorphisms (SNPs), rare copy number variants (CNVs), and rare de novo mutations all contribute substantially to the etiology of psychiatric disorders like schizophrenia (SCZ), bipolar disorder (BD), autism spectrum disorder (ASD), attention-deficit/hyperactivity disorder (ADHD) and others. However, these variants still account for only a small fraction of all the genetic risk for these highly heterogeneous disorders, and much of the heritability remains to be explained. Moreover, the biological 
significance of those disease-associated variants and the true causal factors remain to be discovered.

Which major research areas of psychiatric genetics will yield major breakthroughs in the coming years? Research on deep phenotypes, new phenotypes, epigenetics of large cohorts, cross-disorder analyses, pharmacogenetics, and cellular models will likely bring novel understandings about the etiology and pathology of psychiatric disorders, and better guide development of new treatments. Herein we review a few approaches, including genetics, epigenetics, and molecular and cellular functional studies, and preview their anticipated impacts on the field.

\section{GWAS/WES/WGS WILL CONTINUE TO EXPAND, COVERING MORE DISORDERS, DRUG RESPONSES, PHENOTYPES, AND DIVERSE POPULATIONS}

GWASs will continue to grow in terms of sample size for major psychiatric disorders and will expand to include other heritable disorders. More importantly, researchers designing GWASs should pay attention to population diversity and include under-studied ancestral groups, especially those groups that will help narrow blocks of linkage disequilibrium in the search for causal variants. Pharmacogenetics must catch up with disease GWAS. Gradually, phenotyping will steal center stage from genotyping, but the change will be gradual as phenotyping is more complex and more expensive.

The Psychiatric Genomics Consortium (PGC) currently comprises large-sample studies of SCZ, BD, ADHD, major depression, Tourette Syndrome, obsessive-compulsive disorder, post-traumatic stress disorder, ASD, eating disorder, anxiety, substance use disorder, and Alzheimer's disease. Those studies will continue to grow, leading to discoveries of more common-SNP associations with smaller effects, and polygenic risk scores (PRS) capable of explaining greater variance with increased precision. Several common psychiatric disorders with clear genetic risks have received minimal genetic research efforts to date, including personality disorders [2], sleep disorders [3], conduct disorder [4] and learning disabilities [5].

The population diversity of psychiatric GWASs has been relatively limited to date. The majority of GWASs included only samples from patients of European ancestry. A smaller number of studies involved Asian patients (Han Chinese [6-15], Japanese [16-19], Korean [20], Indian [21,22], Pakistani [23]). Even fewer studies have been completed on populations originating from Africa or South America. From the researcher's point of view, the inclusion of diverse populations in genetic studies can improve the resolution of genetic maps and enhance our ability to identify specific risk genes and regulatory elements in the human genome. In terms of eliminating health disparities, the inclusion of diverse populations in genetic studies is the only way for all humanity to benefit from modern genetics research. 
Pharmacogenetics focuses on identifying genetic factors that influence treatment response, including efficacy and side effects. Although its clinical significance is obvious, pharmacogenetics research into psychiatric diseases is in its early stages, having fewer than 5000 samples tested to date per drug studied. GWASs have been performed on the therapeutic effects and side effects of antipsychotics, selective serotonin reuptake inhibitors, and lithium. As we observed, however, GWASs of drug response to date did not recover any of the top pharmacodynamic candidate genes, such as CYP2D6 and CYP2C19. Future, sufficiently powered GWASs investigating the efficacy and side effects of treatment should better predict appropriate drug choices than current candidate genes. Relating individual genes, gene sets, or polygenic scores to treatment response is the foundation for developing precision medicine. Pharmacogenetic GWASs are more expensive than disease GWASs due to the complexities of the clinical setting, their demand of time and personnel investment for collecting treatment-related data, and the greater degree of patient involvement. The PGC's organizational model of combining data from many small studies could be one of the solutions for building powerful pharmacogenetics datasets. Acquiring data from major medical institutes that have large medical record data collections coupled with biobanks should also be pursued. Meanwhile, current data from small studies are being compiled and interpreted for clinical use by the Clinical Pharmacogenetics Implementation Consortium (CPIC; https://cpicpgx.org/). Researchers should also be aware of an ongoing debate about best practices in establishing the clinical utility of pharmacogenetic findings [24].

Intermediate phenotypes (also called endophenotypes) are quantitative biological traits that are believed to mediate the effects of genes on disorders while having a simpler genetic architecture than the disorders themselves. Such phenotypes have attracted researchers for two major reasons: (1) they can be used to construct a causal framework of complex traits leading to disorders; therefore, they may help to elucidate the biological path between genetic variants and, ultimately, higher-order disease phenotypes; and, (2) they could have a more direct connection to underlying genetics than to the disorder per se. GWASs of intermediate phenotypes could be more powerful than disease GWASs if the simpler genetic architecture of the former is, as hypothesized, associated with larger effect sizes for GWAS SNPs. Consequently, the genetics of intermediate phenotypes could be used to explain GWAS signals (via, for example, Mendelian randomization studies) or to facilitate the identification of disease risk genes.

Several projects have pioneered the collection of intermediate phenotype data from large cohorts. Within the NIMH Research Domain Criteria (RDoC) initiative, the Bipolar and Schizophrenia Network for Intermediate Phenotypes (BSNIP) project has collected deep phenotype data, including cognitive functions, brain imaging, brain electroencephalogram, and eye movement, in patients with BD and SCZ. 
The Philadelphia Neurodevelopmental Cohort [25] has intensive data of cognitive functions. The SUNY Upstate Reward Regulation Project (SUREREG) is studying the genetics of the RDoC Positive Valence System, which comprises several reward-related phenotypes. Brain imaging data has been the focus of the ENIGMA consortium [26].

Several other population- or community-based projects, including the UK Biobank, the Million Veteran Program (MVP), the National Institute of Health (NIH)'s “All of Us”, Adolescent Brain Cognitive Development $(A B C D)$ and 23andMe, should yield important insights into the genetics of many behavioral traits, such as personality, sleep, education, smoking, brain volume and brain connectivity, plus a host of cognitive traits. These big data projects have extensive phenotype data originating from numerous fields. They will offer unique opportunities to study phenotypic and genetic relationships among various disorders and traits. Some of the relationships will be causal, while others will not. Pleiotropic effects will be a common confounder in isolating causal effects. Resolving their relationships will be informative in revealing disease mechanisms.

Developing biobanks coupled with electronic health records (EHR) is an attractive approach to establish large datasets that can be used for studying the biology of various diseases, including psychiatric disorders. Vanderbilt University, Partners HealthCare, and the Mayo Clinic are a few institutes leading the efforts to implement hospital-wide programs. BioVU (https://victr.vumc.org/pub/biovu/) is the Vanderbilt biobank contains medical records of more than 200,000 subjects. The Partners HealthCare Biobank (https://biobank.partners.org/) serves affiliated hospitals in the Boston area. More than 100,000 subjects have been collected by them so far. About 30,000 subjects have been collected by the Mayo Clinic Biobank. Moreover, Mayo Clinic has a separate Biobank specific for bipolar disorder [27]. The Electronic Medical Records and Genomics (eMERGE) network [28], involving ten institutes including Vanderbilt, MGH, and Mayo clinic, has been the major NIH-funded project utilizing medical records for genomic research. ADHD and ASD are part of the project. Otherwise, current EHR-biobank projects do not have a major emphasis on psychiatric disorders yet.

Besides those commonly studied phenotypes described above, we should also adopt and develop new phenotypes that can be measured more efficiently within populations using mobile devices and that have the underlying genetics of large effect size. Mobile device-based digital phenotyping is currently a hot topic with great potential for many creative uses in genetics. Digital phenotyping enables fast collection of large data sets $[29,30]$. New phenotypes can target traits of potentially large genetic effect size. These are two different approaches that would boost statistical power to detect the genetics of new phenotypes. The discovered SNP-associated phenotypes can be used to annotate functions of the SNPs. The phenotype-annotated SNPs can be used to refine the study of disorders. Algorithms like PrediXcan [31] and MetaXcan [32](or 
sPrediXcan) and MulTiXcan [33] will benefit from these data to perform gene-based analyses. On the other hand, genes and pathways associated with intermediate phenotypes will help to dissect mechanism, explain the biology of psychiatric disorders, and many other traits through the technique of phenome-wide association study (PheWAS)[34].

Not all phenotypes are equal in terms of the effect sizes of their genetic factors. Good examples of phenotypes with large effect sizes are molecular phenotypes. Molecular phenotypes, like gene expression and DNA methylation have triumphed in the mapping of regulatory elements. One hundred samples are sufficient to map expression and methylation quantitative traits (eQTLs and mQTLs)[35,36]. Inflammation is another highly interesting phenotype that deserves genetic mapping, given its potential involvement across many psychiatric disorders; nevertheless, only limited studies have been performed so far. One GWAS study of circulating cytokines and growth factors pioneered the mapping of 27 loci [37]. Cellular studies will provide additional promising new phenotypes that will be discussed later in this essay.

Since many more samples will be studied with many more phenotypes including common variants of weak-effect and rare variants of relatively strong effect, these will implicate risk genes and regulatory variants for disease onset, persistence, and treatment response. Polygenic risk scores for traits and disorders will be further refined. But genetics alone will not be enough to accomplish the goals of better disease classification, diagnosis, and treatment; the power of epigenetics and functional studies will be needed to improve the resolution and help uncover mechanisms. And, to fully understand the etiology of psychiatric disorders, the interaction of environmental and genetic risks will require clarification.

Cross-disorder studies will be particularly fruitful in the coming years for psychiatry. It is expected that most psychiatric disorders share some genetic risk variants. The degree of sharing likely defines the degree of shared clinical features among disorders. Cross-disorder studies will not only identify risk genes that contribute to multiple disorders but will also help to identify genes and other genomic features that may differentiate disorders. Cross-disorder genetic and epigenetic studies may eventually redefine current disease classifications, becoming biology-based, objective classifications.

\section{EPIGENETICS OF PSYCHIATRIC DISORDERS WILL BE PURSUED, PROMPTED BY THE EVIDENCE THAT ENVIRONMENTAL FACTORS CHANGE EPIGENETICS AND RISK FOR PSYCHIATRIC DISORDERS}

Epigenetic factors, including DNA methylation, histone modifications, non-coding RNAs, and others, are dynamic regulators of gene expression. They are frequently products of interactions between genetic and environmental factors. Epigenetics is a critical part of the formula to classify disorders and predict risks and treatment response. Normally, 
epigenetic profiling will be performed along with transcriptome profiling, since the chief functional impact of epigenetic modification is thought to alter gene expression.

The epigenotype is the signature of epigenetic marks, the equivalent of DNA genotype. Profiling epigenotypes is more expensive than genotyping and is fraught with technical uncertainties. DNA genotypes are typically biallelic; whereas epigenotypes are mostly quantitative, and their measurement is frequently unstable. Influenced not only by many technical and biological factors, but epigenotypes are also regulated by genetic and environmental factors. DNA methylation is currently the most accessible epigenotype. It costs several hundred to three thousand dollars to assay one sample of one of two types of DNA methylation (5mC and $5 \mathrm{hmC}$ [38], both of which may represent distinct regulatory functions. $5 \mathrm{hmC}$ and non-CpG methylation may carry important regulatory information or case-control differences that have been overlooked in the past because of the limitation of technologies used for profiling.

An epigenome-wide investigation into psychiatric disorders or treatment response is early in development. Longitudinal studies and large consortium projects will be needed to fulfill its promise. Unlike genetics, epigenetics must consider tissue-specificity, age, sex, treatment status, environmental exposure, and other factors. Longitudinal studies of peripheral tissues could track the epigenetic changes associated with different clinical symptoms and treatment stages while serving as a source of useful biomarkers. Unfortunately, most epigenetic studies today are still cross-sectional or case-controlled. Few have been published with two or more time points. The cost of collecting such data is the major limiting factor, as epigenotyping and following-up with patients to collect biospecimens are prohibitively expensive. Instead, a large biobank or collaborative consortium may be necessary to accelerate research progress. Small studies have insufficient statistical power, despite their superior quantitative characteristics of continuous epigenetic data relative to dichotomous genotype data. For this reason, small studies should be designed to follow a consensus design, using standard quality controls to readily combine small data into large analyses.

Gene expression and epigenetic profiles are specified by tissue and cell type. While tissue or cell-type specificity is a big challenge, it also presents an opportunity. As in transcriptome studies, profiling epigenetics in mixed cells could mask differences in subgroups of cells. Single-cell assays are gradually moving to the center stage of research. Nonetheless, applying single-cell assays to large populations still faces a cost barrier. Creative computational and statistical methods could be used to deconvolute the data from mixed cells or gross tissues [39-42], although some details could be lost in deconvolution. Moreover, the validity of those deconvolution methods remains to be proven. The 
compromise between resolution and comprehension in single-cell assays must be balanced, contingent upon specific hypothesis.

Epigenetic markers are not only regulators of gene expression and mediators of high-level phenotypes, including disease diagnoses or behavioral phenotypes; they are also products of genetic-environmental interactions. Medication, drinking, smoking, psychological stress, diet, and many other environmental conditions can provoke epigenetic changes. Integrating epigenetics with genetics and with other -omics may help to resolve some causal contributors to psychiatric disorders.

Environmental factors are known to contribute to epigenetic changes and disease risks. Several prenatal and postnatal factors, associated with alterations of DNA methylation in rodent model experiments and in human patients, may ultimately contribute to disease risk [38]. Alcohol and smoking are the two most studied factors [43-47]. Sex is another very important factor associated with both differential DNA methylation and differential risks for psychiatric disorders [48]. Many environmental factors promote neuroinflammation [49-51], increase oxidative stress [52,53], disturb neuronal development [54,55], and disrupt neuronal networks [55,56]. Such mechanistic models, connecting environmental factors as well as epigenetic and genetic variations to disease risks could be studied in large human populations, including both healthy and affected participants.

Relating peripheral biomarkers to changes in the central nervous system could employ information gleaned from postmortem brains. The PsychENCODE project [57] concentrates a rich resource of brain -omics data for psychiatric studies. It has the advantage of both depth and breadth by collecting multiple types of data from the same individuals while profiling a large number of individuals. PsychENCODE is one of the largest datasets of postmortem brain samples from patients with SCZ, BD, and ASD. After the first set of publications [58-67], the data will be made available to the public for further mining. Much more remains to be discovered from these data.

\section{FUNCTIONAL FOLLOW-UP OF GWAS RESULTS WILL BE PERFORMED IN CELLULAR MODELS}

Identifying GWAS associations is only the first critical step in understanding how a genetic variant can affect the risk for a complex disease. Fine-mapping of associated loci is essential to determine which of several variants in a haplotype block may be truly responsible for the association signal. But identifying causal variation also falls short of having a practical impact, as simply knowing the identity of the variant does not suggest a clinically actionable route, neither a specific gene nor a biological pathway. Thus, the mechanistic study of associated loci is the necessary next step. GWASs alone have limited values without functional follow-up studies. 
Functional follow-up studies of GWAS "hits" (regions of the genome associated with the disease in question) have three primary goals: (1) identifying target genes of disease-associated variants; (2) establishing regulatory relationships, pathways, or networks among all associated variants and genes; and (3) revealing functional impacts of the associated genetic variants and genes on phenotypes at all levels, from molecular and cellular phenotypes to behavioral traits and disorders. These studies pose the greatest challenge for future research. We expect that shortly, cellular models will be the most productive field for discovering target genes, gene networks, and cellular functions relevant to psychiatric illness.

The biological significance of most GWAS signals remains elusive, without even a clear target gene or regulatory sequence for many associated SNPs. Given that an associated SNP could impact the expression of a distant gene, functional studies should begin with those associated SNPs. Brain eQTL and Hi-C data can be used to connect SNPs to their putative target genes. Reporter gene assays, knockdown and overexpression, and CRISPR (clustered regularly interspaced short palindromic repeats) editing can be used to validate the functional impact of specific SNPs and the putative regulatory elements where the SNPs reside [68-71]. Most published research to date typically concludes with an SNP regulating gene expression. Few studies investigate cellular phenotypes, which should be an important focus in the coming years.

Data integration and network construction will generate new knowledge of biological systems, including causal factors, underlying disease risks, and drug responses. Since genetics, multi-omics, and dimensional phenotype data will be generated from large populations, sorting out their relationships and causal connections will be important to resolving the puzzle of disease biology. Computational methods like weighted gene coexpression network analysis (WGCNA) and others have been used to build correlation-based networks. Construction of regulatory networks is a fast-evolving field. Networks can be developed based on quantitative correlations, physical interactions, and literature of biochemical experiments. High-throughput methods will speed up the growth of knowledge about networks. It should be noted that regulatory relationships predicted by in silico analyses need to be experimentally validated. Cellular experiments similar to those done for POU3F2 [59] and DGCR5 [58] will help to construct regulatory networks of gene expression.

Cellular phenotypes are phenotypes that can be measured in cells within a culture or within the living body. Commonly accessible cellular phenotypes include cell morphology, cell functionality, and cell division and differentiation speed, ion channel properties, electrophysiology, cellular responses to drugs or other insults, and interaction with other cells. Cellular phenotypes are another level of intermediate phenotypes that represent functional effects. They reflect biological changes associated with disease risk or with drug response. Cellular phenotypes bridge molecular phenotypes and high-level traits of behavior, brain 
structure, and function. This field requires tremendous investment to reduce cost and increase throughput. The challenges and potential solutions for using cellular models to study psychiatric disorders have been reviewed [72-74]. Molecular profiling coupled with highthroughput techniques that measure cellular phenotypes in vivo, ex vivo, and at the single-cell level, will advance the field significantly.

Identifying cellular phenotypes of genetic variants is different from uncovering a complete molecular mechanism, which requires detailed characterization of each molecule and its physical and biochemical interactions in the process-from the genetic variants to the phenotypes, like electrophysiological measures. Normally, establishing the complete biological mechanism demands extensive experimental steps, including the screening and manipulation of relevant genes and environmental conditions. In contrast, identifying cellular phenotypes associated with genetic variants of both direct and indirect causal relationships would be a reasonable alternative, associating a given cellular phenotype to one gene or genetic variant even while intermediate factors remain unknown. Cellular phenotyping is a readily practical approach for advancing largescale screening, an essential component of genetic studies. Certainly, establishing valid cellular phenotypes and developing high-throughput phenotyping methods still require major investment.

\section{A REMAINING CHALLENGE: GENE NETWORKS AND THE OMNIGENIC MODEL}

Current GWASs clearly show that psychiatric disorders involve hundreds, even thousands, of genes. The omnigenic model even proposed that almost every gene could contribute to disease risks and that "peripheral genes" cumulatively contribute more heritability through trans- effects than "core genes" do through cis-effects [75,76]. Although the omnigenic model is still actively debated, it offers a new perspective about the genetics of complex disorders [77-85]. For the timebeing, it is hard to appreciate the complete biological meaning of genetic findings for all psychiatric disorders. Although only a few specific genes, such as C4, CACNA1C, DRD2, and a few others are the primary focus of many hypotheses, they account for a tiny fraction of disease genetic associations. The same is true for polygenic risk scores and top GWAS signals. We are still "blind men feeling the elephant." Instead, the solution may reside in understanding how proteins coded by implicated genes interact with one another and with regulatory variations identified with GWASs. Recognizing these coded proteins will, in turn, help us to understand their related upstream and downstream biology. Genetic and epigenetic studies, along with cellular experiments, will piece together these networks and improve our understanding of the biological systems underlying psychiatric disorders. 


\section{SUMMARY AND CONCLUSIONS}

In the 1930s, researchers discovered that genetics contribute to psychiatric disorders [86]. Since then, particularly in the last two decades, psychiatric genetics has evolved into an extensive research field and has achieved significant progress. Advanced new technologies and statistical methods made genetic and epigenetic exploration possible, developing more phenotypes in larger populations and in different biological levels. In another ten to twenty years, we will not only have a better understanding of the risk for psychiatric disorders, but we should also have a better understanding of drug-target options for treating them.

Major data resources and consortium projects discussed in this paper are summarized in Table 1.

Table 1. Major biobanks and consortium projects for psychiatric genetics and epigenetics.

\begin{tabular}{|c|c|c|}
\hline Biobanks/Projects & Data & Websites \\
\hline \multicolumn{3}{|l|}{ Psychiatry-specific } \\
\hline $\begin{array}{l}\text { Psychiatric } \\
\text { Genomics } \\
\text { Consortium }\end{array}$ & GWAS of major psychiatric disorders & https://www.med.unc.edu/pgc/ \\
\hline PsychENCODE & Epigenomic of postmortem brains & https://www.nimhgenetics.org/resources/psychencode \\
\hline $\begin{array}{l}\text { Philadelphia } \\
\text { Neurodevelopment } \\
\text { al Cohort }\end{array}$ & Deep phenotyping project & $\begin{array}{l}\text { https://www.med.upenn.edu/bbl/philadelphianeurodeve } \\
\text { lopmentalcohort.html }\end{array}$ \\
\hline $\begin{array}{l}\text { Mayo Clinic Bipolar } \\
\text { disorder biobank }\end{array}$ & Bipolar disorder biobank & $\begin{array}{l}\text { https://www.mayo.edu/research/centers- } \\
\text { programs/bipolar-disorder-biobank }\end{array}$ \\
\hline BSNIP & Deep phenotyping project & http://b-snip.org/ \\
\hline ConLiGen & Consortium on Lithium genetics & http://www.conligen.org/ \\
\hline ENIGMA & $\begin{array}{l}\text { Consortium on neuro-imaging } \\
\text { genetics }\end{array}$ & http://enigma.ini.usc.edu/ \\
\hline \multicolumn{3}{|l|}{ General } \\
\hline GTEx & $\begin{array}{l}\text { Genetics of gene expression } \\
\text { regulation }\end{array}$ & https://gtexportal.org/home/ \\
\hline UK Biobank & $\begin{array}{l}\text { Genetics of diseases and traits of } \\
500 \mathrm{k} \text { UK people }\end{array}$ & https://www.ukbiobank.ac.uk/ \\
\hline MVP & $\begin{array}{l}\text { Genetics of diseases and traits in US } \\
\text { veteran }\end{array}$ & https://www.research.va.gov/mvp/ \\
\hline All of Us & $\begin{array}{l}\text { Research program on environment, } \\
\text { life style and biology of } 1 \text { million } \\
\text { people }\end{array}$ & $\underline{\text { https://allofus.nih.gov/ }}$ \\
\hline $\mathrm{ABCD}$ & $\begin{array}{l}\text { Adolescent Brain Cognitive } \\
\text { Development Study, the largest long- } \\
\text { term study of brain development } \\
\text { and child health in the United States }\end{array}$ & https://abcdstudy.org/ \\
\hline Emerge & EHR-Biobank consortium & https://emerge.mc.vanderbilt.edu/ \\
\hline
\end{tabular}

\section{AUTHOR CONTRIBUTIONS}

CL initiated and prepared the manuscript. SVF and SJG provided new ideas and revised the paper. 


\section{CONFLICTS OF INTEREST}

The authors declare no conflict of interest.

\section{FUNDING}

This work was supported by NIH grants 1U01MH103340, 1U01MH116489 and 1R01MH110920 (to C. Liu), 5R01MH101519 (to SVF and SJG), and 5R01AG054002 (to SJG).

\section{ACKNOWLEDGEMENT}

This is a paper dedicated to Elliot S. Gershon's 79th birthday, to honor his achievements in the field of psychiatric genetics. A small part of his work has been reflected in this paper, primarily as the collaborative research between Elliot S. Gershon and Chunyu Liu. Gershon has been a life-time mentor for Chunyu Liu since 1998. We thank Liz Kuney for helping to edit the text and figure, also thank Chao Chen and Richard Kopp, Chunling Zhang, and three anonymous reviewers for valuable comments.

\section{REFERENCES}

1. Horwitz T, Lam K, Chen Y, Xia Y, Liu C. A decade in psychiatric GWAS research. Mol Psychiatry. 2019;24(3):378-89. doi: 10.1038/s41380-018-0055-z

2. Reichborn-Kjennerud T. The genetic epidemiology of personality disorders. Dialogues Clin Neurosci. 2010;12(1):103-14.

3. Sehgal A, Mignot E. Genetics of sleep and sleep disorders. Cell. 2011;146(2):194-207.

4. Salvatore JE, Dick DM. Genetic influences on conduct disorder. Neurosci Biobehav Rev. 2018;91:91-101.

5. Kovas Y, Haworth CM, Dale PS, Plomin R. The genetic and environmental origins of learning abilities and disabilities in the early school years. Monogr Soc Res Child Dev. 2007;72(3):vii, 1-144.

6. Kalsi G, Euesden J, Coleman JR, Ducci F, Aliev F, Newhouse SJ, et al. GenomeWide Association of Heroin Dependence in Han Chinese. PLoS One. 2016;11(12):e0167388.

7. Yu H, Yan H, Li J, Li Z, Zhang X, Ma Y, et al. Common variants on 2p16.1, 6p22.1 and 10q24.32 are associated with schizophrenia in Han Chinese population. Mol Psychiatry. 2017;22(7):954-60.

8. Yin X, Wineinger NE, Wang K, Yue W, Norgren N, Wang L, et al. Common susceptibility variants are shared between schizophrenia and psoriasis in the Han Chinese population. J Psychiatry Neurosci. 2016;41(6):413-21.

9. Yin CL, Chen HI, Li LH, Chien YL, Liao HM, Chou MC, et al. Genome-wide analysis of copy number variations identifies PARK2 as a candidate gene for autism spectrum disorder. Mol Autism. 2016;7:23.

10. Li Z, Chen J, Xu Y, Yi Q, Ji W, Wang P, et al. Genome-wide Analysis of the Role of Copy Number Variation in Schizophrenia Risk in Chinese. Biol Psychiatry. 2016;80(4):331-7. 
11. Liu C, Saffen D, Schulze TG, Burmeister M, Sham PC, Yao YG, et al. Psychiatric genetics in China: achievements and challenges. Mol Psychiatry. 2016;21(1):4-9.

12. Xia K, Guo H, Hu Z, Xun G, Zuo L, Peng Y, et al. Common genetic variants on 1p13.2 associate with risk of autism. Mol Psychiatry. 2014;19(11):1212-9.

13. Yang X, Lu X, Wang L, Chen S, Li J, Cao J, et al. Common variants at 12q24 are associated with drinking behavior in Han Chinese. Am J Clin Nutr. 2013;97(3):545-51.

14. Lee MT, Chen CH, Lee CS, Chen CC, Chong MY, Ouyang WC, et al. Genomewide association study of bipolar I disorder in the Han Chinese population. Mol Psychiatry. 2011;16(5):548-56.

15. Yang L, Neale BM, Liu L, Lee SH, Wray NR, Ji N, et al. Polygenic transmission and complex neuro developmental network for attention deficit hyperactivity disorder: genome-wide association study of both common and rare variants. Am J Med Genet B. 2013;162B(5):419-30.

16. Koshimizu H, Nogawa S, Asano S, Ikeda M, Iwata N, Takahashi S, et al. Genome-wide association study identifies a novel locus associated with psychological distress in the Japanese population. Transl Psychiatry. 2019;9(1):52.

17. Wu J, Yu P, Jin X, Xu X, Li J, Li Z, et al. Genomic landscapes of Chinese sporadic autism spectrum disorders revealed by whole-genome sequencing. J Genet Genomics. 2018;45(10):527-38.

18. Kanazawa T, Bousman CA, Liu C, Everall IP. Schizophrenia genetics in the genome-wide era: a review of Japanese studies. NPJ Schizophr. 2017;3(1):27.

19. Liu X, Shimada T, Otowa T, Wu YY, Kawamura Y, Tochigi M, et al. Genomewide Association Study of Autism Spectrum Disorder in the East Asian Populations. Autism Res. 2016;9(3):340-9.

20. Cho SC, Yoo HJ, Park M, Cho IH, Kim BN, Kim JW, et al. Genome-wide association scan of korean autism spectrum disorders with language delay: a preliminary study. Psychiatry Investig. 2011;8(1):61-6.

21. Sudesh R, Thalamuthu A, John S, Thara R, Mowry B, Munirajan AK. Replication of GWAS identified miR-137 and its target gene polymorphisms in Schizophrenia of South Indian population and meta-analysis with Psychiatric Genomics Consortium. Schizophr Res. 2018;199:189-94.

22. Prasad S, Bhatia T, Kukshal P, Nimgaonkar VL, Deshpande SN, Thelma BK. Attempts to replicate genetic associations with schizophrenia in a cohort from north India. NPJ Schizophr. 2017;3(1):28.

23. Fatima A, Farooq M, Abdullah U, Tariq M, Mustafa T, Iqbal M, et al. GenomeWide Supported Risk Variants in MIR137, CACNA1C, CSMD1, DRD2, and GRM3 Contribute to Schizophrenia Susceptibility in Pakistani Population. Psychiatry Investig. 2017;14(5):687-92.

24. Zubenko GS, Sommer BR, Cohen BM. On the Marketing and Use of Pharmacogenetic Tests for Psychiatric Treatment. JAMA Psychiatry. 2018;75(8):769-70.

25. Robinson EB, Kirby A, Ruparel K, Yang J, McGrath L, Anttila V, et al. The genetic architecture of pediatric cognitive abilities in the Philadelphia Neurodevelopmental Cohort. Mol Psychiatry. 2015;20(4):454-8. 
26. Thompson PM, Stein JL, Medland SE, Hibar DP, Vasquez AA, Renteria ME, et al. The ENIGMA Consortium: large-scale collaborative analyses of neuroimaging and genetic data. Brain Imaging Behav. 2014;8(2):153-82.

27. Frye MA, McElroy SL, Fuentes M, Sutor B, Schak KM, Galardy CW, et al. Development of a bipolar disorder biobank: differential phenotyping for subsequent biomarker analyses. Int J Bipolar Disord. 2015;3(1):30.

28. Gottesman O, Kuivaniemi H, Tromp G, Faucett WA, Li R, Manolio TA, et al. The Electronic Medical Records and Genomics (eMERGE) Network: past, present, and future. Genet Med. 2013;15(10):761-71.

29. Insel TR. Digital phenotyping: a global tool for psychiatry. World Psychiatry. 2018;17(3):276-7.

30. Reinertsen E, Clifford GD. A review of physiological and behavioral monitoring with digital sensors for neuropsychiatric illnesses. Physiol Meas. 2018;39(5):05TR1.

31. Gamazon ER, Wheeler HE, Shah KP, Mozaffari SV, Aquino-Michaels K, Carroll RJ, et al. A gene-based association method for mapping traits using reference transcriptome data. Nat Genet. 2015;47(9):1091-8.

32. Barbeira AN, Dickinson SP, Bonazzola R, Zheng J, Wheeler HE, Torres JM, et al. Exploring the phenotypic consequences of tissue specific gene expression variation inferred from GWAS summary statistics. Nat Commun. 2018;9(1):1825.

33. Barbeira AN, Pividori MD, Zheng J, Wheeler HE, Nicolae DL, Im HK. Integrating Predicted Transcriptome From Multiple Tissues Improves Association Detection. PLoS Genet. 2019;15(1):e1007889. doi: 10.1371/journal.pgen.1007889

34. Denny JC, Ritchie MD, Basford MA, Pulley JM, Bastarache L, Brown-Gentry K, et al. PheWAS: demonstrating the feasibility of a phenome-wide scan to discover gene-disease associations. Bioinformatics. 2010;26(9):1205-10.

35. Liu C. Brain expression quantitative trait locus mapping informs genetic studies of psychiatric diseases. Neurosci Bull. 2011;27(2):123-33.

36. Liu C. QTL Mapping of Molecular Traits for Studies of Human Complex Diseases. In: Shugart YY, editor. Applied Computational Genomics. Dordrecht (The Netherlands): Springer; 2012. p. 61-82.

37. Ahola-Olli AV, Wurtz P, Havulinna AS, Aalto K, Pitkanen N, Lehtimaki T, et al. Genome-wide Association Study Identifies 27 Loci Influencing Concentrations of Circulating Cytokines and Growth Factors. Am J Hum Genet. 2017;100(1): 40-50.

38. Liu C, Jiao C, Wang K, Yuan N. DNA Methylation and Psychiatric Disorders. Prog Mol Biol Transl Sci. 2018;157:175-232.

39. Wang N, Hoffman EP, Chen L, Chen L, Zhang Z, Liu C, et al. Mathematical modelling of transcriptional heterogeneity identifies novel markers and subpopulations in complex tissues. Sci Rep. 2016;6:18909.

40. Gaujoux R, Seoighe C. CellMix: a comprehensive toolbox for gene expression deconvolution. Bioinformatics. 2013;29(17):2211-2. 
41. Newman AM, Liu CL, Green MR, Gentles AJ, Feng W, Xu Y, et al. Robust enumeration of cell subsets from tissue expression profiles. Nat Methods. 2015;12(5):453-7.

42. Shen-Orr SS, Gaujoux R. Computational deconvolution: extracting cell typespecific information from heterogeneous samples. Curr Opin Immunol. 2013;25(5):571-8.

43. Bonsch D, Lenz B, Fiszer R, Frieling H, Kornhuber J, Bleich S. Lowered DNA methyltransferase (DNMT-3b) mRNA expression is associated with genomic DNA hypermethylation in patients with chronic alcoholism. J Neural Transm. 2006;113(9):1299-304.

44. Guidotti A, Dong E, Gavin DP, Veldic M, Zhao W, Bhaumik DK, et al. DNA methylation/demethylation network expression in psychotic patients with a history of alcohol abuse. Alcohol Clin Exp Res. 2013;37(3):417-24.

45. Philibert RA, Plume JM, Gibbons FX, Brody GH, Beach SR. The impact of recent alcohol use on genome wide DNA methylation signatures. Front Genet. 2012;3:54.

46. Satta R, Maloku E, Zhubi A, Pibiri F, Hajos M, Costa E, et al. Nicotine decreases DNA methyltransferase 1 expression and glutamic acid decarboxylase 67 promoter methylation in GABAergic interneurons. Proc Natl Acad Sci U S A. 2008;105(42):16356-61.

47. Shenker NS, Ueland PM, Polidoro S, van Veldhoven K, Ricceri F, Brown R, et al. DNA methylation as a long-term biomarker of exposure to tobacco smoke. Epidemiology. 2013;24(5):712-6.

48. Xia Y, Dai R, Wang K, Jiao C, Zhang C, Xu Y, et al. Sex-differential DNA methylation and associated regulation networks in human brain implicated in the sex-biased risks of psychiatric disorders. Mol Psychiatry. 2019. doi: 10.1038/s41380-019-0416-2

49. Alrouji M, Manouchehrinia A, Gran B, Constantinescu CS. Effects of cigarette smoke on immunity, neuroinflammation and multiple sclerosis. J Neuroimmunol. 2019;329:24-34.

50. Kelley KW, Dantzer R. Alcoholism and inflammation: neuroimmunology of behavioral and mood disorders. Brain Behav Immun. 2011;25(Suppl 1):S13-20.

51. Monnig MA. Immune activation and neuroinflammation in alcohol use and HIV infection: evidence for shared mechanisms. Am J Drug Alcohol Abuse. 2017;43(1):7-23.

52. Albano E. Alcohol, oxidative stress and free radical damage. Proc Nutr Soc. 2006;65(3):278-90.

53. van der Vaart H, Postma DS, Timens W, ten Hacken NH. Acute effects of cigarette smoke on inflammation and oxidative stress: a review. Thorax. 2004;59(8):713-21.

54. Semick SA, Collado-Torres L, Markunas CA, Shin JH, Deep-Soboslay A, Tao R, et al. Developmental effects of maternal smoking during pregnancy on the human frontal cortex transcriptome. Mol Psychiatry. 2018. doi: 10.1038/s41380-018-0223-1

55. Tateno M, Saito T. Biological studies on alcohol-induced neuronal damage. Psychiatry Investig. 2008;5(1):21-7. 
56. Lithari C, Klados MA, Pappas C, Albani M, Kapoukranidou D, Kovatsi L, et al. Alcohol affects the brain's resting-state network in social drinkers. PLoS One. 2012;7(10):e48641.

57. Psych EC, Akbarian S, Liu C, Knowles JA, Vaccarino FM, Farnham PJ, et al. The PsychENCODE project. Nat Neurosci. 2015;18(12):1707-12.

58. Meng Q, Wang K, Brunetti T, Xia Y, Jiao C, Dai R, et al. The DGCR5 long noncoding RNA may regulate expression of several schizophrenia-related genes. Sci Transl Med. 2018;10(472). doi: 10.1126/scitranslmed.aat6912

59. Chen C, Meng Q, Xia Y, Ding C, Wang L, Dai R, et al. The transcription factor POU3F2 regulates a gene coexpression network in brain tissue from patients with psychiatric disorders. Sci Transl Med. 2018;10(472). doi: 10.1126/scitranslmed.aat8178

60. Wang D, Liu S, Warrell J, Won H, Shi X, Navarro FCP, et al. Comprehensive functional genomic resource and integrative model for the human brain. Science. 2018;362(6420). doi: 10.1126/science.aat8464

61. Gandal MJ, Zhang P, Hadjimichael E, Walker RL, Chen C, Liu S, et al. Transcriptome-wide isoform-level dysregulation in ASD, schizophrenia, and bipolar disorder. Science. 2018;362(6420). doi: 10.1126/science.aat8127

62. Zhu Y, Sousa AMM, Gao T, Skarica M, Li M, Santpere G, et al. Spatiotemporal transcriptomic divergence across human and macaque brain development. Science. 2018;362(6420). doi: 10.1126/science.aat8077

63. Li M, Santpere G, Imamura Kawasawa Y, Evgrafov OV, Gulden FO, Pochareddy S, et al. Integrative functional genomic analysis of human brain development and neuropsychiatric risks. Science. 2018;362(6420). doi: 10.1126/science.aat7615

64. Amiri A, Coppola G, Scuderi S, Wu F, Roychowdhury T, Liu F, et al. Transcriptome and epigenome landscape of human cortical development modeled in organoids. Science. 2018;362(6420). doi: 10.1126/science.aat6720

65. An JY, Lin K, Zhu L, Werling DM, Dong S, Brand H, et al. Genome-wide de novo risk score implicates promoter variation in autism spectrum disorder. Science. 2018;362(6420). doi: 10.1126/science.aat6576

66. Kozlenkov A, Li J, Apontes P, Hurd YL, Byne WM, Koonin EV, et al. A unique role for DNA (hydroxy)methylation in epigenetic regulation of human inhibitory neurons. Sci Adv. 2018;4(9):eaau6190.

67. Rajarajan P, Borrman T, Liao W, Schrode N, Flaherty E, Casino C, et al. Neuron-specific signatures in the chromosomal connectome associated with schizophrenia risk. Science. 2018;362(6420). doi: 10.1126/science.aat4311

68. Duan J, Shi J, Fiorentino A, Leites C, Chen X, Moy W, et al. A rare functional noncoding variant at the GWAS-implicated MIR137/MIR2682 locus might confer risk to schizophrenia and bipolar disorder. Am J Hum Genet. 2014;95(6):744-53.

69. Forrest MP, Zhang H, Moy W, McGowan H, Leites C, Dionisio LE, et al. Open Chromatin Profiling in hiPSC-Derived Neurons Prioritizes Functional Noncoding Psychiatric Risk Variants and Highlights Neurodevelopmental Loci. Cell Stem Cell. 2017;21(3):305-18.e8. 
70. Huo Y, Li S, Liu J, Li X, Luo XJ. Functional genomics reveal gene regulatory mechanisms underlying schizophrenia risk. Nat Commun. 2019;10(1):670.

71. Sekar A, Bialas AR, de Rivera H, Davis A, Hammond TR, Kamitaki N, et al. Schizophrenia risk from complex variation of complement component 4. Nature. 2016;530(7589):177-83.

72. Brennand KJ, Simone A, Tran N, Gage FH. Modeling psychiatric disorders at the cellular and network levels. Mol Psychiatry. 2012;17(12):1239-53.

73. Falk A, Heine VM, Harwood AJ, Sullivan PF, Peitz M, Brustle O, et al. Modeling psychiatric disorders: from genomic findings to cellular phenotypes. Mol Psychiatry. 2016;21(9):1167-79.

74. Vadodaria KC, Amatya DN, Marchetto MC, Gage FH. Modeling psychiatric disorders using patient stem cell-derived neurons: a way forward. Genome Med. 2018;10(1):1.

75. Boyle EA, Li YI, Pritchard JK. An Expanded View of Complex Traits: From Polygenic to Omnigenic. Cell. 2017;169(7):1177-86.

76. Liu X, Li YI, Pritchard JK. Trans Effects on Gene Expression Can Drive Omnigenic Inheritance. Cell. 2019;177(4):1022-34.e6.

77. Wray NR, Wijmenga C, Sullivan PF, Yang J, Visscher PM. Common Disease Is More Complex Than Implied by the Core Gene Omnigenic Model. Cell. 2018;173(7):1573-80.

78. Boyle EA, Li YI, Pritchard JK. The Omnigenic Model: Response from the Authors. J Psychiatry Brain Sci. 2017;2(5):S8.

79. Cox NJ. Comments on Pritchard Paper. J Psychiatry Brain Sci. 2017;2(5):S5.

80. Faraone SV. The Omnigenic Model: Implications for Psychiatric Genetics. J Psychiatry Brain Sci. 2017;2(5):S1.

81. Franke B. What's in a Name: the “Omnigenic” Model as a Point of Departure for Polygenic Psychiatric Disorders. J Psychiatry Brain Sci. 2017;2(5):S7.

82. Gershon ES, Alliey-Rodriguez N. Comment on: An Expanded View of Complex Traits: From Polygenic to Omnigenic. J Psychiatry Brain Sci. 2017;2(5):S3.

83. He X. Comment on: An Expanded View of Complex Traits: From Polygenic to Omnigenic. J Psychiatry Brain Sci. 2017;2(5):S2.

84. Liu C. A Case for Core Genes. J Psychiatry Brain Sci. 2017;2(5):S6.

85. McMahon F. Casting a Shadow of Doubt over the GWAS Parade. J Psychiatry Brain Sci. 2017;2(5):S4.

86. Slater E. The Inheritance of Manic-depressive Insanity: (Section of Psychiatry). Proc R Soc Med. 1936;29(8):981-90.

How to cite this article:

Liu C, Faraone SV, Glatt SJ. Psychiatric Genetics, Epigenetics, and Cellular Models in Coming Years. J Psychiatry Brain Sci. 2019;4:e190012. https://doi.org/10.20900/jpbs.20190012 